Check for updates

Cite this: RSC Adv., 2017, 7, 22998

\title{
MSCs on an acellular dermal matrix (ADM) sourced from neonatal mouse skin regulate collagen reconstruction of granulation tissue during adult cutaneous wound healing
}

\begin{abstract}
Maosheng Chen, (D) Ying Jin, Xue Han, Ning Wang, Xiaoyuan Deng* and Hanping Liu*
Full-thickness cutaneous wound repair in adults usually leads to non-regenerative healing, which produces disorganized and non-functional fibrotic tissues. Mesenchymal stem cells (MSCs) on acellular dermal matrix (ADM) scaffolds are currently believed to be a promising strategy for wound healing improvement. Extracellular matrix (ECM) is aware of playing a pivotal role in wound healing, and changing the properties of the ECM is therefore expected to alter repair outcomes. Inspired by the analogous regeneration process in foetal skin with minimal or no scar tissue formation, in this study, ADM scaffold sourced from 1 day old mouse skin is introduced. Its influence on collagen reconstruction of granulation tissue during adult fullthickness wound healing is revealed and is compared to that from 20 week old mouse skin. Advanced nonlinear optical two-photon fluorescence (TPF) and second-harmonic generation (SHG) imaging were used to intravitally and three-dimensionally monitor the activities of MSCs and formation of granulation tissue. Dynamic changes of collagen content in granulation tissue were analyzed from aspects of synthesis and degradation. The associated collagen proteins, type I and type III collagen (Col-I and Col-III) and matrix matalloproteinase-13 (Mmp-13) were assessed at the transcriptional, translational and deposition levels. The results showed that there were significant differences in remodelling characteristics as a consequence of ADM properties. One-day old ADM + MSC treatment induced up-regulation of Col-III expression, downregulation of Col-I and also down-regulation of Mmp-13. Accordingly, 1 day ADM + MSC treatment caused higher type III collagen deposition and a higher ratio of Col-III/Col-l in the granulation tissue. ADM derived from 1 day old skin ECM was superior to ADM derived from 20 week old skin ECM, caused enhanced angiogenesis, down regulation of TGF- $\beta 1$ and promoted re-epithelization and faster, more constructive, and compositionally appropriate formation of granulation tissue. These results suggest that ADM derived from 1 day old skin ECM is a favourable biomaterial for adult wound healing.
\end{abstract}

Received 20th February 2017

Accepted 20th April 2017

DOI: $10.1039 / \mathrm{c} 7 \mathrm{ra02125a}$

rsc.li/rsc-advances

\section{Introduction}

Adult full-thickness cutaneous wound repair usually leads to formation of a scar composed of disorganized and nonfunctional fibrotic tissues instead of normal dermal tissue. ${ }^{1}$ Controlling repair towards regenerative healing with little or no scar formation is the subject of intense investigation for adult skin regeneration. ${ }^{2}$

Wound repair is a complex process orchestrated by a series of cells, multiple biological factors, and critical dynamic interactions between cells and the surrounding extracellular matrix (ECM). ${ }^{3}$ ECM is not just pretty fibrils. ${ }^{4}$ During the past decade, researchers have developed a more intricate understanding of wound biology and have recognized the importance of non-

MOE Key Laboratory of Laser Life Science, College of Biophotonics, South China Normal University, Guangzhou, Guangdong, China. E-mail: xydeng12@126.com; liuhp@scnu.edu.cn cellular ECM components. Far beyond providing structural support, the ECM provides cells a dynamic biophysical, biomechanical and biochemical micro-environment (a niche) and exerts tailored biological activities to manipulate cell behaviour. ${ }^{5}$ Thus, ECM derived from intact mammalian tissues has attracted increased attention as bio-scaffold for application during various tissue engineering/regenerative therapies. ${ }^{6} \mathrm{ECM}$ bio-scaffold has demonstrated its ability to alter the default cutaneous wound healing response in regulating proinflammatory, scarring events toward a more constructive remodelling response of a site appropriate formation of functional tissues. ${ }^{7}$ Hence, changing the characteristics of ECM bioscaffolds and thus modifying the inductive micro-environment is anticipated to be a novel strategy for developing interventions that achieve desirable cutaneous wound healing: ${ }^{8}$

Characterized by site- and tissue-specific regeneration and very minimal scar tissue formation ${ }^{9}$ the healing of foetal skin offers hints that scar tissue formation is not inevitable. The 
underlying mechanism of foetal skin healing presents distinct difference from that of wound healing in adults, which is associated with selected pro-inflammatory events, distinct matrix reconstruction, desirable epithelialization and other events. ${ }^{10}$ The defined wound healing response of foetal skin is believed to be intrinsic to the foetal skin ECM rather than the foetal environment. ${ }^{11}$ Differences in collagen type, organization, bio-mechanics and other properties have been noted in foetal and adult skin ECM. Collagen in foetal skin ECM is mainly type III, while the adult skin ECM contains primarily type I. Additionally, the ECM of adult skin is "stiffer" than the ECM of foetal skin. ${ }^{12,13}$ Therefore, an ECM bio-scaffold derived from neonatal or newborn skin is presumed to be a more suitable biomaterial than ECM derived from adult skin and may promote favourable wound healing by providing a foetal ECM-approaching microenvironment.

ECM bio-scaffolds sourced from different animal ages do have demonstrated disparate impacts on wound healing. Small intestinal submucosa (SIS)-ECM scaffolds derived from different pig ages $(3,12,26$, and $>52$ weeks) applied for abdominal wall musculature wound healing in rat have revealed that the SIS-ECM from younger animals induced a more constructive, site appropriate, tissue remodeling response with respect to macrophage phenotypes, vascularisation, innervation and myogenesis than scaffolds derived from older animals. ${ }^{\mathbf{1 4}}$ Acellular dermal matrix (ADM), mainly from human or animal skin dermal ECM, has emerged as a promising biomaterial and has recently been used successfully to promote full-thickness cutaneous wound repair. ${ }^{15,16}$ Our previous research has directly demonstrated that ADM from neonatal or newborn mouse skin has a favourable influence than that from adult skin on cutaneous wound healing. ${ }^{17}$

The beneficial effect of transplanting exogenous mesenchymal stem cells (MSCs) on wound healing has been clearly observed in a variety of animal models and in clinical cases. ${ }^{18}$ Although the mechanisms underlying the actions of MSCs during the wound healing process are unclear, the ECM is clearly involved and provides a defined micro-environment that regulates MSC activities, including survival, proliferation, differentiation, migration and paracrine function. ${ }^{19}$ Thus, seeding MSCs onto optimized ADMs is considered a promising therapeutic strategy for promoting scar-less cutaneous wound healing. It is expected that the interactions of MSCs with the designed ADM bio-scaffold may actively guide and control the MSCs behaviours and ultimately influence the outcomes of wound healing.

The reconstruction of granulation tissue is one of the most vital periods for restoration of damaged tissues during both early and late phases of wound healing. ${ }^{20}$ Granulation tissue is a collagen rich connective tissue generally formed within 5-12 days after injury. Thus, the study of collagen synthesis, degradation, and deposition is essential for understanding the reconstruction of granulation tissues. The purpose of this study is to reveal how MSCs on different ADM scaffolds, derived from neonatal skin and adult skin, influence the collagen rebuilding process in granulation tissue, and identify an ADM bio-scaffold with properties favourable for adult wound healing. Such studies may also provide clues for the further exploration of the mechanisms underlying these processes.

\section{Materials and methods}

\subsection{Preparation of acellular dermal matrix (ADM) scaffold}

Skins obtained respectively from 20 week old male Balb/c mice and 1 day old male Balb/c mice were used to be made for ADM scaffolds. The mice were anesthetized by the pelltobarbitalum natricum through the intraperitoneal injection. The dorsal hair was shaved and pieces of full-thickness skin were harvested from the center of dorsal. The skin tissues were treated with 0.25\% Dispase (Aoboxing, Beijing, China) at $4{ }^{\circ} \mathrm{C}$ for $48 \mathrm{~h}$ to separate the epidermis. Then the processed skin tissues were immersed in PBS mixed with $0.3 \%$ Triton X-100, 0.25\% deoxycholate sodium and $0.02 \%$ EDTA under continuous vibration at $37{ }^{\circ} \mathrm{C}$ for another $48 \mathrm{~h}$ in order to remove the cellular components from the extracellular matrix. Next, the decellularized scaffolds were treated with a fat digestion solution of chloroform and methanol $(\mathrm{v} / \mathrm{v}=1: 1)$ for $1 \mathrm{~h}$ and washed off the solution with PBS. Finally, the scaffolds were cut to several circular sections in a diameter of $7 \mathrm{~mm}$. Then the scaffolds were sterilized in $70 \%$ ethanol for overnight and washed three times with sterile PBS before use.

\subsection{Assessment of mechanical properties of ADMs}

The mechanical properties of 1 day ADM and 20 week ADM were measured using a uni-axial load testing equipment (ELF 3200, Bose Corporation, U.S.A) at the room temperature of $25{ }^{\circ} \mathrm{C}$. Before the test, the ADM samples $(2.0 \mathrm{~mm}$ of width $\times 14 \mathrm{~mm}$ of length $\times 0.06 \mathrm{~mm}$ of thickness for 1 day ADM and $0.46 \mathrm{~mm}$ of thickness for 20 week ADM) were clamped for axial tensile testing $(n=5)$ at a ramp force of $0.1 \mathrm{~N} \mathrm{~min}^{-1}$ for 1 day ADM and $0.5 \mathrm{~N} \mathrm{~min}^{-1}$ for 20 week ADM. The stress was monitored as a function of strain and the elastic modules was obtained by analysing the linear region of the resulting stress-strain curves.

\subsection{Seeding of MSCs on ADM and MSCs proliferation assay}

C57BL/6 mouse bone marrow mesenchymal stem cells (mBMSCs, MUCMX-01101, GFP-tagged) were purchased from Cyagen Biosciences, Inc. (Guangzhou, China), and cultured in OriCellTM mouse MSC Growth Medium (MUCMX-90011, Cyagen Biosciences, Inc.) in a humidified incubator (Thermo Scientific Forma 3110, Thermo Fisher Scientific, Inc.) at $37{ }^{\circ} \mathrm{C}$ with $5 \% \mathrm{CO}_{2}$. Round pieces of ADM scaffolds (7 mm in diameter) were acquired via biopsy punches and placed in a 48-well plate (one piece per well) with the papillary dermis side facing upward. ADM scaffolds were incubated overnight in fresh MSC Growth Medium, and then $1 \times 10^{5}$ MSCs were seeded onto the scaffolds and co-cultured for one more day before being transplanted onto animal models.

To understand the proliferation of MSCs on the ADM scaffold, the number of MSCs after 2, 5 and 7 co-culture days with ADM scaffold were checked and immunofluorescence staining of GFP was performed as the standard protocol. Seeding onto the ADM scaffolds, most of the MSCs attached to the ADM 
scaffolds after $6 \mathrm{~h}$ incubation. On the 2nd, 5th and 7th incubation day, the co-cultured MSC + ADM mixtures were gently washed to remove non-adherent and dead cells and then gone through the standard immunofluorescence stain procedures. The number of MSCs was quantified by the intensity of GFP and the number of nuclei. The fluorescence intensity of GFP was analysed using Image Pro Plus software.

\subsection{SEM (scanning electron microscope) imaging of ADM}

ADM scaffolds were fixed in $2.5 \%$ glutaraldehyde at $4{ }^{\circ} \mathrm{C}$ for at least $24 \mathrm{~h}$. Scaffolds were then dehydrated by immersion in an ascending ethanol series $(25 \%, 50 \%, 75 \%$, and $100 \%)$, treated twice with isoamyl acetate, critical point dried and sputtercoated with a $30 \mathrm{~nm}$ gold layer. Samples were imaged using a scanning electron microscope (Zeiss Ultra 55, Carl Zeiss, Jena, Germany) in order to show the collagen organization and construction in the ADM scaffolds.

\subsection{Wound model and surgical procedure}

All procedures were performed in accordance with the National Institute of Health (NIH) guidelines for care and use of laboratory animals, and with approval of the Ethical Committee for Animal Experiments of South China Normal University. 10 week old Balb/c male mice were purchased from Laboratory Animal Center of Southern Medical University and used as the receptor model. Animals were anesthetized with an intraperitoneal injection of pelltobarbitalum natricum. Once the mice were anesthetized, the hair of each mouse was shaved and removed by hair removal cream. After hair removed, the bared skin was sterilized with $75 \%$ ethanol prior to surgery. A $7 \mathrm{~mm}$ fullthickness wound was created by a $7 \mathrm{~mm}$ biopsy punch (Harris Uni-Core ${ }^{\mathrm{TM}}$ ). The wounded mice were divided into three groups and were separately treated with 1 day ADM + MSCs, 20 week $\mathrm{ADM}+\mathrm{MSCs}$ and untreated as control. After the treatment was completed, Comfeel transparent dressings (Coloplast, Beijing, China) were used to cover the wound sites to prevent further catching, biting and bacterial infection.

\subsection{Histological and immunofluorescent analysis}

Wound dorsal skin samples were harvested at designated post operation day (POD). Samples were fixed in 10\% formalin at room temperature for at least $24 \mathrm{~h}$, embedded in paraffin and cut into $5 \mu \mathrm{m}$ thick longitudinal sections. The sections were deparaffinized, re-hydrated and washed with $\mathrm{ddH}_{2} \mathrm{O}$ and stained with hematoxylin and eosin (H\&E). For immunofluorescence staining, sections were deparaffinized and heatmediated antigen retrieval were performed with citrate buffer $(\mathrm{PH}=6.0)$. Then blocked by incubation with peroxide block and normal goat serum (Abcam, UK). Samples were then incubated overnight with primary antibodies against type I collagen (Col-I, 1 : 50, Abcam), type III collagen (Col-III, $1: 80$, Abcam), platelet endothelial cell adhesion molecule-1 (CD-31, $1: 100$, Abcam), transforming growth factor- $\beta 1$ (TGF- $\beta 1,1: 50$, Santa Cruz), green fluorescence protein (GFP, $1: 100$, Abcam) and $\alpha$-alphaSMA $\left(\alpha-S M A, 1: 100\right.$, Abcam) at $4{ }^{\circ} \mathrm{C}$. After washing, the samples incubated with the secondary antibody: goat anti- rabbit IgG (Alex Fluor ${ }^{\circledR} 488$ conjugated, $1: 200$, CST), and goat anti-rat IgG (Alex Fluor ${ }^{\circledR} 555$ conjugated, 1 : 500, CST). Image acquisition was performed using a Zeiss confocal microscope (Zeiss, Oberkochen, Germany).

\subsection{Protein isolation and western blotting analysis}

To collect protein lysates from wound in vivo, wound skin was harvested at 7 PODs and flash-frozen in liquid $\mathrm{N}_{2}$. Skin was minced and added to radioimmuno precipitation assay buffer (50 mM Tris, $150 \mathrm{mM} \mathrm{NaCl,} \mathrm{0.1 \%} \mathrm{SDS,} \mathrm{0.5 \%} \mathrm{Na} \mathrm{deoxycholate,}$ $1 \%$ NP-40) containing protease inhibitors (Roche, Indianapolis, IN). The tissue was homogenized, sonicated for 10 minutes, and frozen at $-80{ }^{\circ} \mathrm{C}$. Total protein was measured using the Bradford assay, and $10 \mu \mathrm{g}$ of protein was run on $8-15 \%$ Tris-glycine gels before being transferred to nitrocellulose membranes. Membrane were blocked with blocking buffer (5\% non-fat dry milk, $10 \mathrm{mM}$ Tris, $100 \mathrm{mM} \mathrm{NaCl,} \mathrm{0.1 \%} \mathrm{Tween-20)} \mathrm{and} \mathrm{probed}$ with the following primary antibodies: rabbit polyclonal to type I collagen ( $1: 500$, Abcam), rabbit polyclonal to type III collagen (1 : 1000, Abcam), rabbit polyclonal to Mmp-13 (1: 800, Abcam), mouse monoclonal to GADPH (1:800 Abcam), and then secondary antibodies $(1: 1000$, peroxidase-conjugated $\alpha$-mouse IgG, $\alpha$-rabbit IgG, KPL). Western blots were visualized via DAB Horseradish Peroxidase Color Development Kit according to the manufacture's protocol (Beyotime, Beijing, China).

\subsection{Quantitative real-time PCR}

Tissue sample were disrupted with a rotor-star homogenizer. Total RNA was isolated from tissue homogenates using Trizol reagent (TaKaRa, Dalian, China). First strand cDNA was synthesized using the PrimeScript ${ }^{\circledR}$ RT reagent Kit with gDNA Eraser (TaKaRa, Dalian, China). The specific prime sequences for cloning and expression were described in the Table 1 . The quantitative real-time PCR was conducted using SYBR® Premix Ex Taq II (Tli RNaseH Plus) (TaKaRa, Dalian, China) in a typical $20 \mu \mathrm{l}$ PCR mixture that include $10 \mu \mathrm{l}$ of SYBR ${ }^{\circledR}$ Premix Ex Taq ${ }^{\mathrm{TM}}$ II, 1-5 $\mu \mathrm{l}$ of template cDNA, and $0.4 \mu \mathrm{M}$ of each PCR primer. Cycling conditions were $95{ }^{\circ} \mathrm{C}$ for $30 \mathrm{~s}$, followed by 40 cycles at $95{ }^{\circ} \mathrm{C}$ for $5 \mathrm{~s}, 60^{\circ} \mathrm{C}$ for $30 \mathrm{~s}$, and melt curve stage was $95{ }^{\circ} \mathrm{C}$ for $15 \mathrm{~s}, 60{ }^{\circ} \mathrm{C}$ for $1 \mathrm{~min}$, and $95{ }^{\circ} \mathrm{C}$ for $15 \mathrm{~s}$ according to the protocol. The relative level of gene expression was run on the Applied Bio-systems 7500/7500 Fast Real-Time PCR System using the $2^{-\Delta \Delta C_{\mathrm{t}}}$ method.

Table 1 The primers of genes analysed in RT-qPCR

Gene type Oligo name Sequence $\left(5^{\prime}\right.$ to $\left.3^{\prime}\right)$

Type I collagen

(Col-I)

Type III collagen (Col-III)

Type 13 matrix metalloproteinase

(Mmp-13)
Forward Reverse Forward Reverse Forward

Reverse
-TGA CTG GAA GAG CGG AGA GT-GAC GGC TGA GTA GGG AAC AC-AGG TTC TCC TGG TGC TGC T-GGA TGC CCA CTT GTT CCA T-CCA TCC CGT GAC CTT ATG TT- 


\subsection{Intravital imaging of granulation tissue}

The intravital images were done by the same multi-photon microscopic imaging system as that in our previous studies. This imaging system for second-harmonic generation (SHG) imaging and two-photon fluorescence (TPF) imaging consists of a commercial LSM 710 NLO confocal microscope (Zeiss, Jane, Germany) coupled with a femtosecond Ti:sapphire laser (Chameleon Vision II, Coherent, Santa Clara, CA, USA). Ti:sapphire laser offers an excitation pulse laser with wavelengths tunable from $680 \mathrm{~nm}$ to $1080 \mathrm{~nm}$. This combined TPF/SHG imaging system carries out high-contrast multi-mode imaging, SHG imaging for collagen and fluorescence imaging for living cells simultaneously. The optical emission signals of SHG and TPF are collected in a backward geometry.

After the mice $(n=4)$ were anesthetized at 7 PODs, the wound tissues were harvested and immersed in PBS buffer for next imaged by TPF and SHG microscopy using the stack scan mode. The samples were laid on cover-slips against by fascia layer. The excitation wavelength was set to $820 \mathrm{~nm}$ for SHG imaging of collagen and $850 \mathrm{~nm}$ for the living GFP-labeled MSCs. The respectively signals were received by two independent channels SHG (400-420 nm) and GFP-fluorescence (500-600 nm). A water-immersion $63 \times$ C-Apochromat objective $(\mathrm{NA}=1.2)$ was used. The step size along $z$ axis was $1 \mu \mathrm{m}$ for 3D imaging.

\subsection{Statistical analysis}

All data are represented as means \pm standard deviation (SD) with at least three independent replicates. To determine significance between groups, comparisons were made using Student's $t$-tests. Analyses of multiple groups were performed using one-way ANOVA with Bonferroni's post-test with GraphPad Prism version 8.0 for windows (Graph-Pad Software, La Jolla, CA). For all statistical tests, the 0.05 level of confidence was accepted for statistical significance.
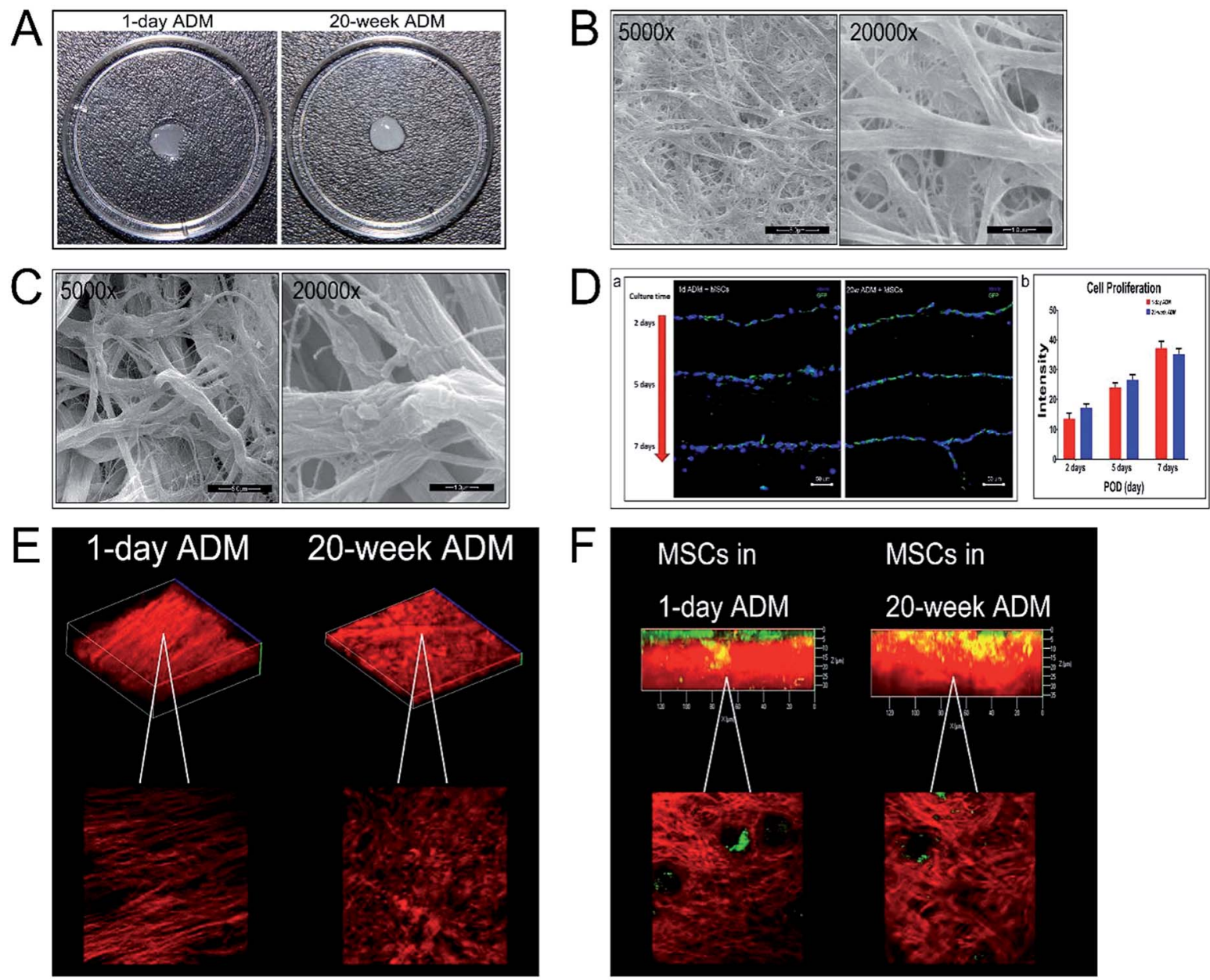

Fig. 1 Characterization of the two ADMs (A) the appearance of the ADM after decellularization treatment in a $30 \mathrm{~mm}$ culture dish. 1 day ADM (left); 20 week ADM (right). (B) Scanning electron microscopy (SEM) of 1 day ADM at two different magnifications of 5000× (left) and 20 000 $\times$ (right) and the extra high tension is $20 \mathrm{kV}$ (C) SEM of 20 week ADM at the magnification of $5000 \times$ (left) and $20000 \times$ (right) and the extra high tension is $20 \mathrm{kV}$ (D) MSC proliferation on the two ADMs after 2,5 and 7 incubation days (D-a) and statistical analysis of the fluorescence intensity (D-b). (E) Second harmonic imaging (SHG) of the collagen (red) characterization in 1 day ADM (left) and 20 week ADM (right). (F) Second harmonic imaging (SHG) of the MSCs (eGFP-tagged) seeded on 1 day ADM (left) and 20 week ADM (right) after $24 \mathrm{~h}$ culture time. MSCs (green) and collagen (red). Scale bar $=5.0 \mu \mathrm{m}(5000 \times)$ and $1.0 \mu \mathrm{m}(20000 \times)$. 


\section{Results}

\subsection{The characteristics of ADM scaffolds}

We prepared the ADM scaffolds by degrading fresh skin tissue from mice. The ADM scaffolds were porcelain white and transparent. They were soft and restorable for tissue engineering. The ADM scaffolds derived from 1 day and 20 week old mice are shown in Fig. 1A. The average thickness of the 1 day ADM scaffolds was $56 \pm 3 \mu \mathrm{m}$ and that of the 20 week ADM scaffolds was $460 \pm 2.3 \mu \mathrm{m}$. These ADM scaffolds were composed of collagen fibrils and lacked cells. The microstructure of these two ADM scaffolds was visualized by SEM at magnifications of $5000 \times$ and $20000 \times$ and are shown in Fig. $1 \mathrm{~B}$ and C, respectively. As shown in Fig. 1D-a, the MSCs seeded onto the two ADM scaffolds proliferated as the incubation times went by. Statistical analysis demonstrated that the proliferation of MSCs on the two different ADM scaffolds made no significant difference (Fig. 1D-b). SHG microscopy revealed the shape, orientation, and density of collagen fibres within the ADM scaffolds (Fig. 1E). The diameters of collagen bundles in the 1 day and 20 week ADM scaffolds were $0.62 \pm 0.12 \mu \mathrm{m}$ and $1.5 \pm$ $0.2 \mu \mathrm{m}$, respectively. The inter-activities of MSCs in the two ADM scaffolds were monitored using a multiphoton microscopic imaging system (Fig. 1F). The red fluorescence represented the collagen composition in the ADM scaffolds and the green fluorescence showed the MSCs seeded on the scaffolds.

The mechanical properties of these two ADM scaffolds were significantly different, as shown in Fig. 2A. Comparison of the diameters of the collagen bundles is shown in Fig. 2A-a and their thicknesses are shown in Fig. 2A-b. In Fig. 2B, two stressstrain curves (stress being a measure of force intensity, given as force per or oriented area while strain being a normalized measure of deformation indicating changes in lengths or angles

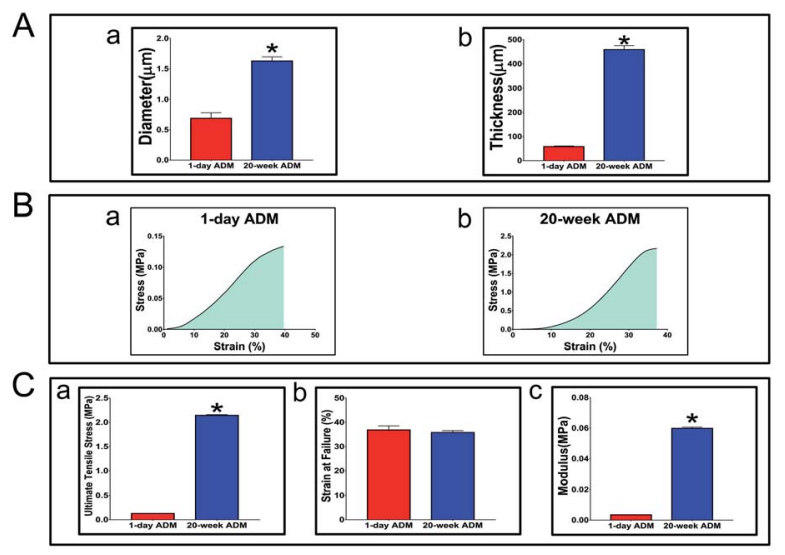

Fig. 2 Mechanical properties of the two ADMs (A) average diameter of the collagen fibers in the ADMs was measured from SEM images (A-a) and average thickness of the two ADMs involved in this study were displayed in (A-b). (B) The stress-strain curves of 1 day ADM and 20 week ADM were shown in (B-a) and (b). (C) Ultimate tensile stress of 1 day ADM was significant lower than that in 20 week ADM (C-a) and also the same difference in the modulus $(\mathrm{C}-\mathrm{C})$. On the other hand, strain at failure did not show significant difference $(C-b)$. Values shown are means \pm SD. $* p<0.05, * * p<0.01, * * * p<0.001$. within a material) were displayed separately and the corresponding specific properties were calculated as shown in Fig. 2C. The ultimate tensile stress is shown in Fig. 2C-a, strain at failure is shown in Fig. 2C-b, and Young's modulus indicating stiffness (how much stress changes when strained) is shown in Fig. 2C-c. Compared to the 20 week ADM scaffold, the 1 day ADM scaffold had a lower ultimate tensile stress (strength) and Young's modulus, but there was no significant difference in strain at failure.

\subsection{One-day ADM with MSCs promoted wound repair in vivo}

MSCs and ADM scaffolds were implanted into wounded tissue, and the host animals were euthanized at 1, 3, 5, 7, 14, 21 and 30 PODs to determine outcomes and perform histology. Generally, we observed murine skin wound healing through macroscopic imaging, but macroscopic images can not reveal deep tissue regeneration. In this study, we observed the deep wound area in histologically stained sections. Samples were embedded in paraffin and sectioned at $5 \mu \mathrm{m}$ increments. Sections were then stained with haematoxylin and eosin (H\&E). All stained sections were consecutively imaged by taking 5-6 pictures at a magnification of $5 \times$. Next, we spliced these consecutive images together into a whole histochemical view of the samples. Fig. 3A displayed the time course of wound healing of the two experimental groups, 1 day ADM + MSCs and 20 week ADM + MSCs, from 1 POD to 30 PODs. The appearances of the wound outcomes are shown in Fig. 3B, and the generation of hair follicles is shown in Fig. 3C. At 21 PODs, newly generated hair follicles were only found in the 1 day ADM + MSCs group, and abundant hair follicles were found in this group at 30 PODs. This result indicated that 1 day ADM + MSCs enhanced the generation of skin appendages. To better analyse the repair, we measured the thickness of the epidermis and the diameter of the wounds (Fig. 3D-a and b). Previous research has found that as repair progresses, the epidermis becomes thinner and epidermal thickness is generally used to evaluate outcome. As shown in Fig. 3D-a, at 14 PODs, 21 PODs and 30 PODS, the epidermis of the 20 week ADM + MSCs group was approximately three times thicker than the epidermis in the 1 day ADM + MSCs group. After 30 PODs, the 1 day ADM + MSCs group's epidermal thickness resembled the original epidermal thickness, in other words, the 1 day ADM + MSCs group was more efficient than the 20 day ADM + MSCs group at regenerating tissue that closely resembles the original normal tissue sample. There were significant differences in epithelial thickness between the two experimental groups, 1 day ADM + MSCs and 20 week ADM + MSCs. Similar changes occurred in the diameter of wound closure. As shown in Fig. 3Db, from 1 to 30 PODs, the 1 day ADM + MSCs group exhibited more rapid wound healing than the 20 week ADM + MSCs group. In the first few days, there were no significant differences in wound closure between the two groups, but at 7 PODs and later, wound healing in the 1 day ADM + MSCs group was significantly different than healing in the 20 week ADM + MSCs group. In conclusion, at the histomorphologic level, 1 day ADM + MSCs treatment can accelerate wound healing and restore tissue better than the 20 week ADM + MSCs treatment. 

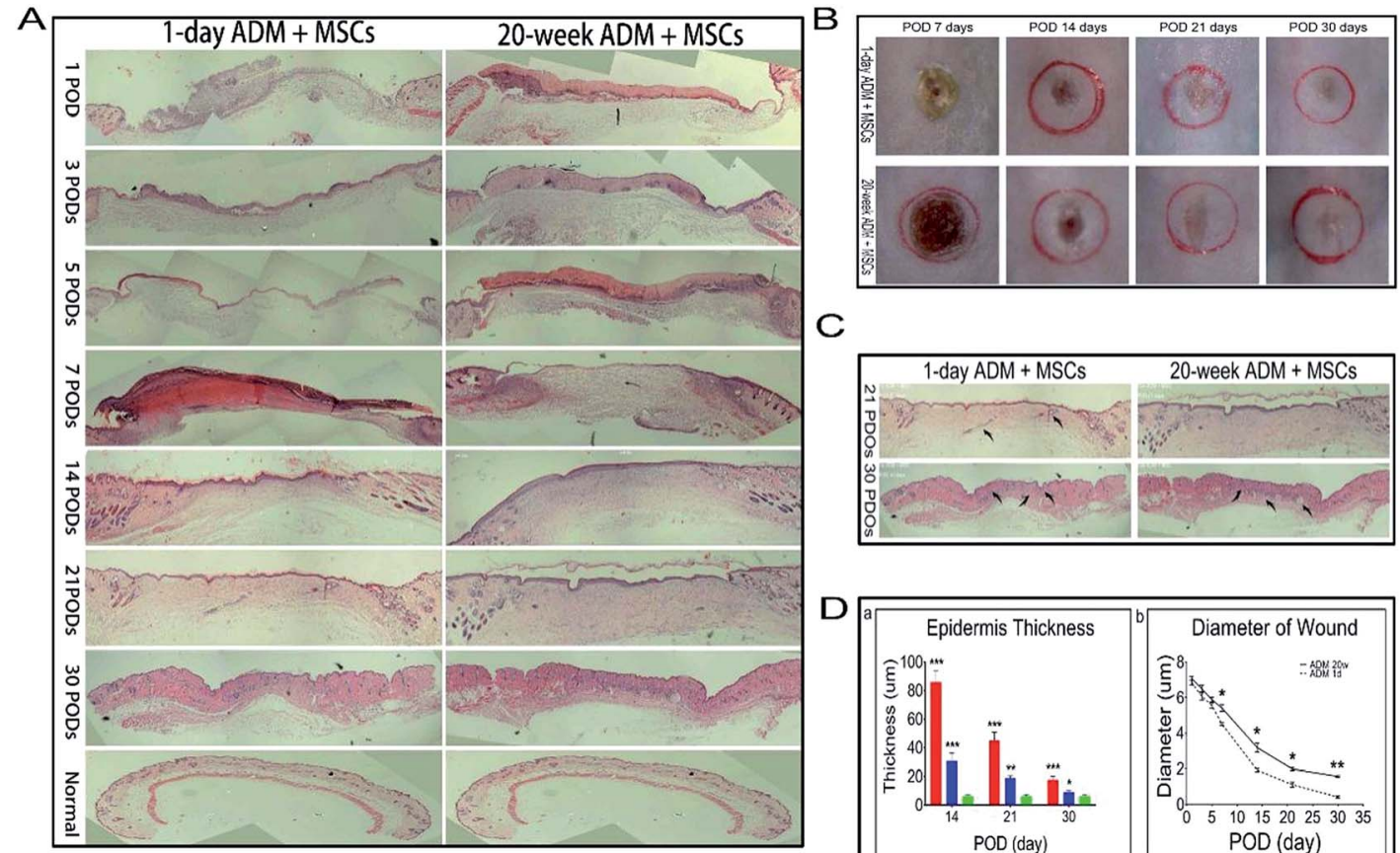

C
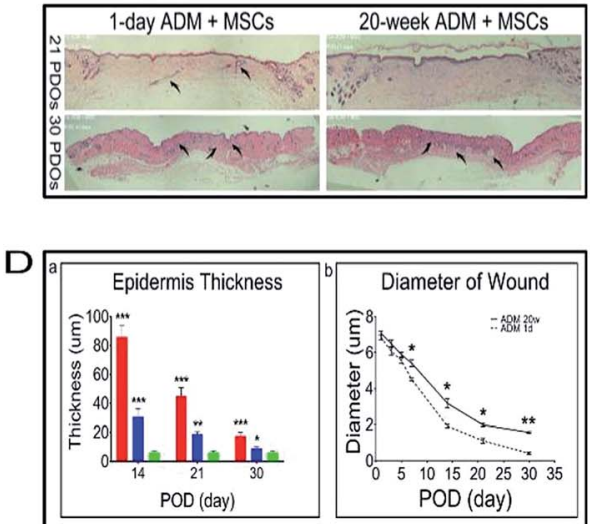

Fig. 3 Improvements of 1 day ADM + MSCs treatment on adult wound healing. (A) Seamless images of H\&E stained whole histological wound sites. 1 day ADM + MSCs group (left) and 20 week ADM + MSCs (right). (B) Macroscopic observation of adult skin wounds after treatment with 1 day ADM + MSCs (up) and 20 week ADM + MSCs (down). Representative images of the wounds on 7, 14, 21, and 30 PODs. (From left to right) (C) The generation of hair follicle in the wound repair was displayed in (C). It showed that at 21 PODs the hair follicle was first found in 1 day ADM + MSCs group but not in 20 week ADM + MSCs group. It indicated that 1 day ADM + MSCs treatment enhanced the hair follicle generation. The hair follicle was pointed by the black arrow. (D) The measurement of the epidermis thickness in wound sites at 14, 21, and 30 PODs and the diameter of wound of each treatment over time (D-a). Quantification of epidermis thickness showed that 1 day ADM + MSCs (blue column) had a thinner epidermis reconstruction than 20 week ADM + MSCs (red column) and was closer to normal skin (green column) (D-b). Measurements of wound diameter displayed 1 day ADM + MSCs (dashed line) significantly improved wound closure compared to 20 week ADM + MSCs (solid line) at 7, 14 , 21 , and 30PODs. Values shown are means \pm SD. ${ }^{*} p<0.05, * * p<0.01, * * * p<0.001$.

\subsection{Evaluation of angiogenesis response and TGF- $\beta 1$ expression}

The angiogenic response, an important step in the wound healing process for severe injuries was also analysed in this study. Alpha-smooth muscle actin ( $\alpha$-SMA) which is used to identify mature vessel stabilized with smooth muscle cells and CD-31 protein which is used to identify new-formed vessel were both detected in wound sections at 7 PODs, which is the time when granulation tissue generally formed (Fig. 4A and B). The results showed that there were fewer mature and new-formed vascular vessels in wounds treated with 20 week ADM + MSCs. The blood vessel density, both mature and new-formed vessel, was significantly higher in the 1 day ADM + MSCs group compared to the 20 week ADM + MSCs group. As demonstrated in Fig. $4 \mathrm{D}-\mathrm{a}$ and $\mathrm{b}$, the $\alpha$-SMA density of the 1 day ADM + MSCs group was approximately four times higher than the density in the 20 week ADM + MSCs group and the CD-31 density was about two times. Moreover, we found that the mature vessel diameter was larger in the 1 day ADM + MSCs group (Fig. 4D-c). The above data indicated that 1 day ADM + MSCs promoted the recruitment of endothelial cells to the wound area and enhanced vessel growth during the healing process.

TGF- $\beta 1$, which is found to play an important role in wound healing process and highly expressed TGF- $\beta 1$ could cause a scar formation, was analysed through immunofluorescence staining in the meantime (shown in Fig. 4C). The results showed that the expression of TGF- $\beta 1$ in the 20 week ADM + MSCs group was approximately 1.4 times higher than the expression in the 1 day $\mathrm{ADM}+\mathrm{MSCs}$ group (Fig. 4D-d), which indicated that the treatment of 1 day ADM + MSCs could be a favorable strategy conducive to relieve the scar formation in wound healing and promote a scar-less healing outcome.

\subsection{Intravital imaging analysis of newly formed granulation tissue}

The remodeling and reconstruction of granulation tissues during wound healing are generally considered to influence the succeeding stages of the wound healing process. Using TPF/ SHG imaging, the 3-D structure of granulation tissue in the two groups was revealed as shown in Fig. 5A. Two major qualities, the thickness of granulation tissue and migratory ability of MSCs, were analysed. In Fig. 5B, the red fluorescence is representative of the collagen composition and the green fluorescence is representative of the cell composition. In the 20 week $\mathrm{ADM}+\mathrm{MSCs}$ group, there was a gap positioned on the left, and the green fluorescence was positioned mostly on top. The above two characteristic phenomena revealed that in the 20 week ADM + MSCs group, the ADM transplant was partially degraded, but 

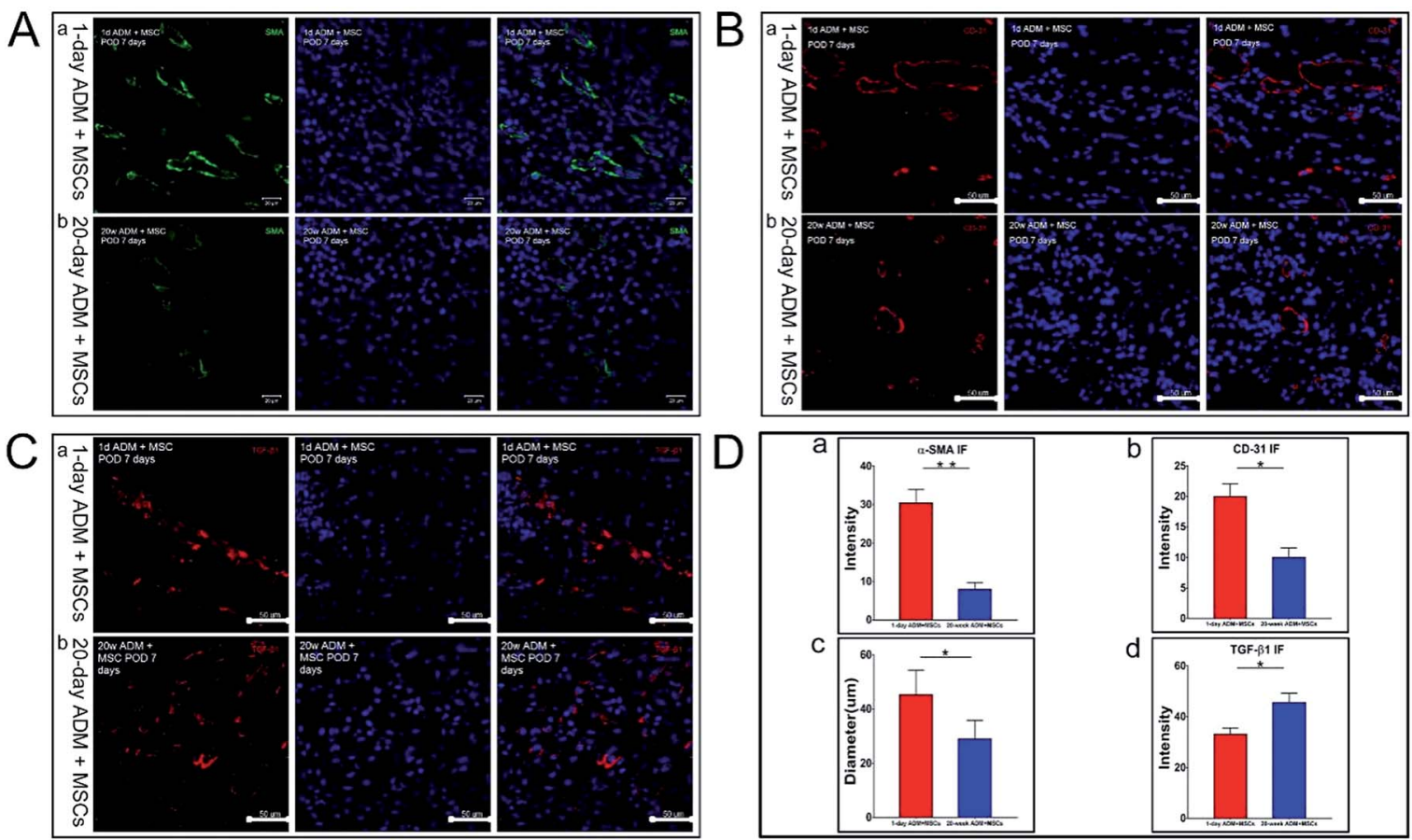

Fig. 4 One-day ADM + MSCs accelerated the angiogenesis in the granulation tissue compared to 20 week ADM + MSCs and down-regulation of TGF- $\beta 1$. (A) Showed the confocal microscopic observation of the mature vessels immunostained with $\alpha$-SMA in granulation tissue sections at 7 PODs in 1 day ADM + MSCs (A-a) and 20 week ADM + MSCs (A-b). Identification of new-formed vessels immunostained with CD-31 protein in 1 day ADM + MSCs and 20 week ADM + MSCs were correspondingly shown in (B-a and B-b). (C) Showed the expression of TGF- $\beta 1$ in 1 day ADM + MSCs group ( $C-a)$ and 20 week ADM + MSCs group (C-b). The intensity of $\alpha$-SMA, CD-31, mature vessel diameter and intensity of TGF- $\beta 1$ were processed and the corresponding statistical analyses were listed in (D-a-d). Abbr, $\alpha$-SMA, alpha-smooth muscle actin. Values shown are means \pm SD. ${ }^{*} p<0.05,{ }^{* *} p<0.01$, scale bar $=20 \mu \mathrm{m}(\mathrm{A}), 50 \mu \mathrm{m}$ (B and C).

the 1 day ADM was totally degraded. We further measured the thickness of the newly formed granulation tissue and found that the 1 day $\mathrm{ADM}+$ MSCs generated thicker granulation tissue $(40 \pm 6 \mu \mathrm{m})$ than the 20 week ADM + MSCs group $(28 \pm 3 \mu \mathrm{m})$, as shown in Fig. 5D-a. The cell migration ability of the transplants is shown in Fig. 5C. In the 1 day ADM + MSCs group (Fig. 5C-a), the green fluorescence was distributed uniformly in the granulation tissue; however, in the 20 week ADM + MSCs group (Fig. 5C-b), it was distributed mostly on top. We calculated the cell intensity in images scanned at $5 \mu \mathrm{m}$ steps on the $z$ axis. Measurements of total cell intensity, as shown in Fig. 5D-b, indicated that there was a higher cell survival rate in the 1 day ADM + MSCs group. The heat map displayed in Fig. 5D-c revealed cells distributed from 15 to $45 \mu \mathrm{m}$ in the 1 day ADM + MSCs group and cells distributed from 40 to $55 \mu \mathrm{m}$ in the 20 week ADM + MSCs group. These results suggest that treatment with 1 day ADM + MSCs resulted in thicker granulation tissue and supported better cell migration within the granulation tissue, indicating that the seeded MSCs actively participate in wound healing.

\subsection{Quantitative real-time PCR analysis of collagen and collagenase mRNA expression}

Collagen is the dominant structural component of mammalian connective tissue. Better regeneration of collagen significantly influences cell differentiation and cytokine secretion and thus accelerates the wound healing process. Previous studies demonstrated that in foetal wound healing, the regenerated skin had a high ratio of Col-III to Col-I. In this section, the expression of type I collagen (Col-I), type III collagen (Col-III) and matrix metalloproteinase-13 (Mmp-13), factors which control the synthesis, degradation and final features of collagen in granulation tissue, were quantified. Quantitative real-time PCR was performed to analyse the expression of the above three target genes. The primers used for quantitative PCR are listed in Table 1. As previously described, the 2(-Delta Delta C (T)) method $\left(2^{-\Delta \Delta C_{\mathrm{t}}}\right)$ was used for the collection and statistical analysis of the data. In Fig. 6A-C, a comparison of the expression levels of the three specified genes within the two experimental groups was shown. Fig. 6A-a showed that type I collagen (Col-I) gene expression was not significantly different between these two groups. Col-III gene expression in the 1 day ADM + MSCs group was four times higher than that in the 20 week $\mathrm{ADM}+\mathrm{MSCs}$ group, as shown in Fig. 6A-b. In contrast, the expression of the type I and type III collagenase, $M m p-13$ in the 20 week ADM + MSCs group, was over ten times higher than that in the 1 day $\mathrm{ADM}+\mathrm{MSCs}$ group, as shown in Fig. 6A-c. The results revealed that at the transcriptional level, Col-III mRNA accumulated faster, while collagenase Mmp-13 mRNA accumulated slower in the 1 day ADM group relative to the 20 week ADM group.

To better compare the transcription of Col-I and Col-III between these two groups, we performed an intra-group analysis of these two genes in the 1 day ADM and 20 week ADM 

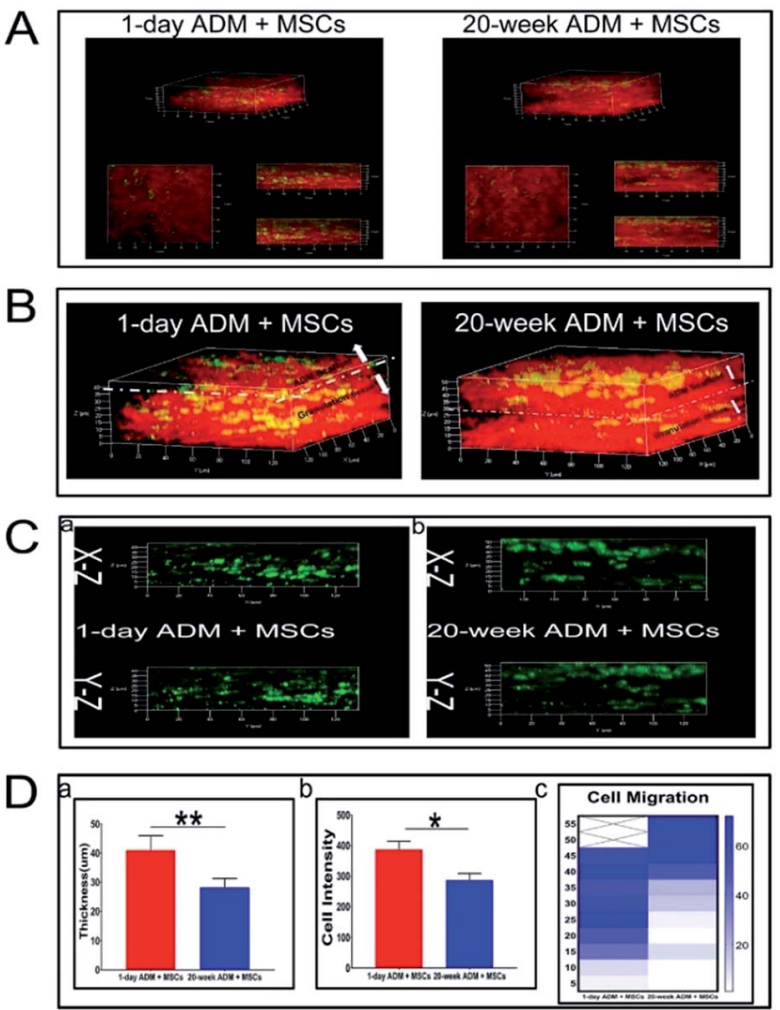

Fig. 5 SHG imaging analysis of regenerated granulation tissue at 7 PODs. The regeneration of granulation tissue was significant upregulated in 1 day ADM + MSCs group. The 3-D dimensional structure of granulation tissue was revealed by SHG imaging (A). In the 1 day $A D M+M S C s$ group the granulation tissue generated more composition compared to 20 week ADM + MSCs group (B). Moreover, after seeded on the 1 day ADM + MSCs, the MSCs showed a better ability to migrate among the wound bed and had a higher survival rate (C). All the results found were analysed in (D). The content of granulation tissue in the 1 day ADM + MSCs group was significantly different with the 20 week ADM + MSCs group as shown in (D-a). And the cell content was calculated by the green fluorescence intensity (D-b) and the heat map was displayed (D-c) values shown are means $\pm S D . * p<$ $0.05, * * p<0.01$.

groups, as shown in Fig. 6B-a and b, respectively. This analysis revealed that the ratio of $\mathrm{Col}-\mathrm{III} / \mathrm{Col}-\mathrm{I}$ in the 1 day and 20 day groups was 4.67 and 1.22 , respectively. Therefore, during wound healing, the 1 day ADM has the potential to induce high levels of transcription of type III collagen in granulation tissue.

\subsection{Western blotting to detect the translation of collagen and collagenase proteins}

In addition to analysing expression at the transcriptional level, we performed a series of western blotting experiments to analyse the synthesis and degradation of type I collagen (Col- $\mathrm{I}$ ), type III collagen (Col-III) and matrix metalloproteinase-13 (Mmp-13) in granulation tissue at the level of protein translation. The western blotting experiments were performed as previously described and the protein expression of Col-I, Col-III and Mmp-13 was quantified. Western blotting results for the two types of collagen protein are shown in Fig. 7A-a and b and for matrix metalloproteinase-13 in Fig. 7A-c. GADPH was used as the reference gene. The untreated control group was indicated by the letter A, the 1 day ADM + MSCs group was indicated by the letter $\mathrm{B}$, and the 20 week ADM + MSCs group was indicated by the letter C. In Fig. 7B-a, we found no significant difference in the level of collagen I protein translation between the two experimental groups, which was similar to the results obtained by RT-qPCR. The protein levels of type III collagen, as shown in Fig. 7B-b, were four times higher in the 1 day ADM + MSCs group than in the 20 week ADM + MSCs group, which was also consistent with the RT-qPCR results. Meanwhile, as shown in Fig. 7B-c, the expression of $\mathrm{Mmp}-13$ protein in the 20 week ADM + MSCs group was 2.5 times higher than that in the 1 day ADM + MSCs group. Therefore, we concluded that compared to the 20 week ADM + MSCs group, the 1 day ADM + MSCs group synthesized more type III collagen protein and had less collagen degradation, resulting in more rapid type III collagen accumulation during the formation of granulation tissue.

A further intra-group comparison of these two types of collagen proteins correlated with the above RT-qPCR results. In Fig. 7C-b, we noted that Col-III protein expression in the 1 day $\mathrm{ADM}+\mathrm{MSCs}$ group was two times higher than Col-I protein expression. These results indicated the higher levels of type III collagen synthesized in granulation tissue. In contrast, as shown in Fig. 7C-c, Col-I protein expression in the 20 week ADM + MSCs group was two times higher than that of Col-III protein expression.

\subsection{Analysis of the deposition of collagen in the ECM with immunofluorescence}

Collagen is the major component of the extracellular matrix (ECM). It forms the skeleton of the extracellular matrix which supplies the living environment for cells. Collagen deposited within the ECM constructs a 3-D environment by cross-linking with other components. To completely reveal the fate of collagen during wound healing, a quantitative analysis of collagen deposited in the ECM was performed using immunofluorescence.

Images of stained slides were captured and processed and are shown in Fig. 8 with continuous fluorescent images of the 1 day ADM + MSCs group in Fig. 8A and the 20 week ADM + MSCs group in Fig. 8B. We analysed the fluorescent intensity of these images using Image Pro Plus software. In Fig. 8C and D, we presented a comparison of the collagen I and collagen III staining. The content of type I collagen in the 1 day ADM + MSCs group was 3.1-fold less than that of the 20 week ADM + MSCs group. Similar to the western blotting results, the staining intensity of type III collagen in the 1 day ADM + MSCs group was 1.5-fold higher than expression in the 20 week ADM + MSCs group. An intra-group comparison of the two collagen types is shown in Fig. 8E and F. Fig. 8E revealed that in the 1 day ADM + MSCs group, there was no significant difference between the two types of deposited collagen. Fig. $8 \mathrm{~F}$ showed that the deposited type I collagen was 3.5 times higher than type III collagen in the 20 week ADM + MSCs group. 

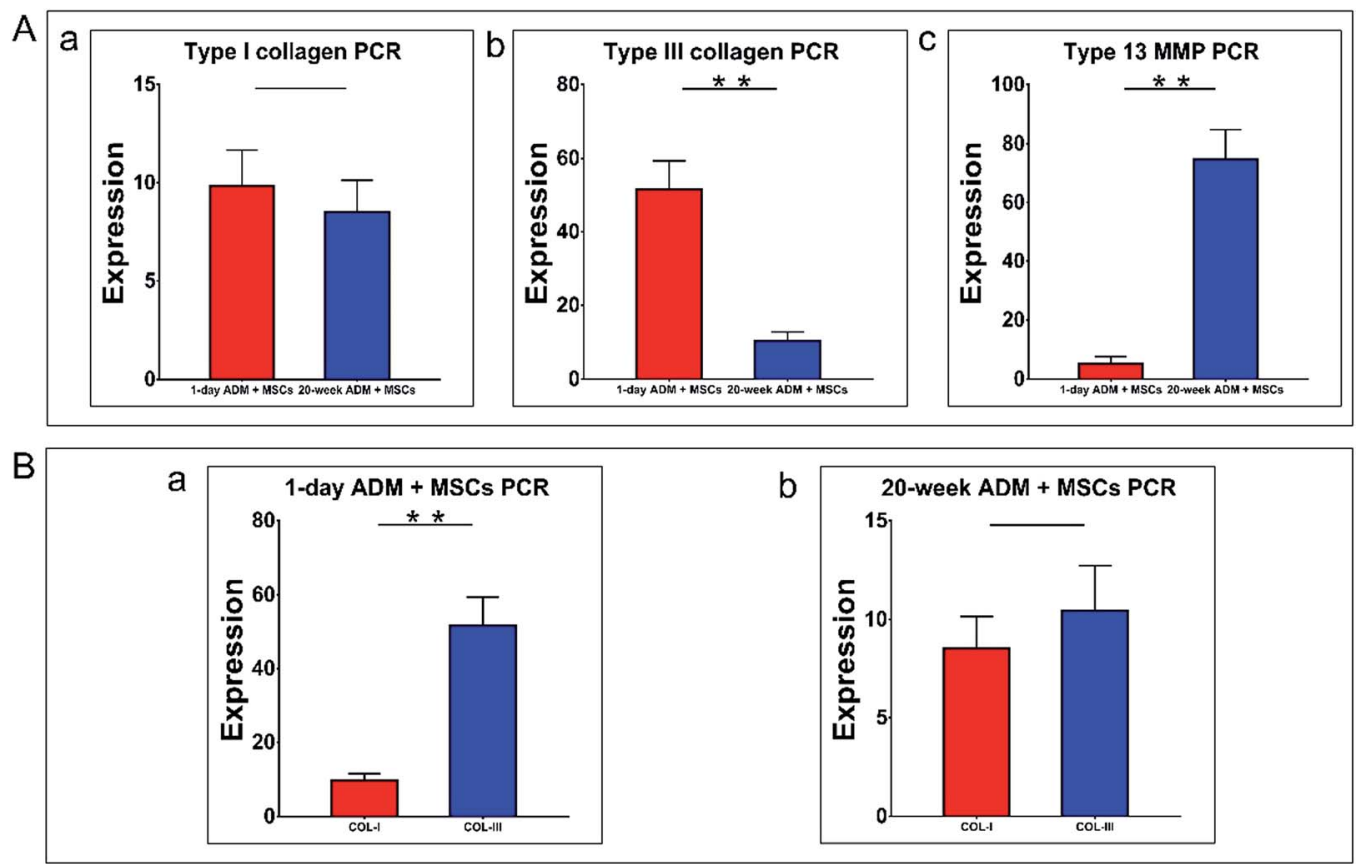

Fig. 6 RT-qPCR comparing expression of three genes between the experimental groups. (A) The comparison between the two groups. In (A-a), no significant difference was observed in Col-I gene expression. Col-III expression in the 1 day ADM + MSCs group was four times higher than in the 20 week ADM + MSCs group (A-b). Mmp-13 gene expression in the 20 week ADM + MSCs was more than 10 times higher than in the 1 day ADM + MSCs group (A-C). (B) Intra-group comparison of Col-I and Col-III gene expression. (B-a) Showed that Col-III expression in the 1 day ADM + MSCs group was about six times higher than the expression of Col-I. There was no significant difference between the two genes in the in 20 week ADM + MSCs group (B-b). The expression levels were normalized to those observed in the control group, as required by the $2^{-\Delta \Delta C_{t}}$ method, and represented as the means \pm SD $(n=4)$. Abbreviations: Col-I, type I collagen; Col-III, type III collagen; Mmp-13, type 13 matrix metalloproteinase; values shown are means $\pm \mathrm{SD}$. $* p<0.05, * * p<0.01$.

We conclude that during the formation of granulation tissue, there was higher expression and deposition of type III collagen, lower expression and deposition of type I collagen and less collagen degradation in the 1 day ADM + MSCs group than in the 20 day + MSCs group. This resulted in a high ratio of collagen type III to I in the 1 day ADM + MSCs group.

\section{Discussion}

The ECM, as a biomaterial scaffold derived from mammalian tissue, has been successfully applied to improve wound healing. ${ }^{21}$ Previous evidence has shown that effective cutaneous wound healing is characterized by the stimulation of reepithelization, promotion of angiogenesis, rapid formation of granulation tissue and reformation of a structure that mimics the original tissue. ${ }^{22}$

MSCs seeded on 1 day ADM promoted re-epithelization, functional skin appendage generation and revascularization. The 1 day ADM + MSCs treatment reconstructed the newborn epithelium at 7 PODs, which was earlier than the 20 week ADM + MSCs treatment. At the end of the re-epithelization process, treatment with 1 day ADM + MSCs rebuilt a thinner epithelial layer, which was approximately the thickness of the normal epithelium. The epithelium during the period of granulation tissue formation in the 1 day ADM + MSCs group was also noted to be thinner than the epithelium in the 20 week ADM + MSCs group, implying that during granulation, a thin epithelium will tend to grow in a way that mimics formation of a normal mature epithelium. The 1 day ADM + MSCs group was also found to promote the earlier generation of functional skin appendages. In this group, the generation of hair follicles began at 21 PODs, and by 30 PODs, hair follicles were abundant, indicating completion of the wound healing process and the formation of a newly functional reconstructed skin. ${ }^{23}$ Similarly, enhancement was found during the revascularization stage. It was observed that at 7 PODs, the density of new-formed and mature vascular vessels were higher in the newly formed granulation tissue in the 1 day ADM + MSCs group than in the 20 week ADM + MSCs group, suggesting that treatment with 1 day ADM + MSCs more efficiently promotes angiogenesis. Moreover, the expression of TGF- $\beta 1$ was found that it was much less in the 1 day ADM + MSCs group than that in the 20 week ADM + MSCs group. In fetal, expression of TGF- $\beta 1$ has been confirmed to be lower during wound healing process compared to that in adult. ${ }^{24}$ Also, a high-expression of TGF- $\beta 1$ is supposed to result in a scar formation. ${ }^{25}$ Our results indicated that under the treatment of 1 day ADM + MSCs, the underlying wound healing process is more similar to that in fetal, thus the strategy of 1 day ADM + MSCs has the potential to relieve the scar formation and promote a scarless wound healing.

The dynamic changes that occurred during the synthesis of collagen in granulation tissue formed during wound healing following treatment with 1 day ADM + MSCs or 20 week ADM + MSCs were imaged in 3-D using the combined TPF/SHG 

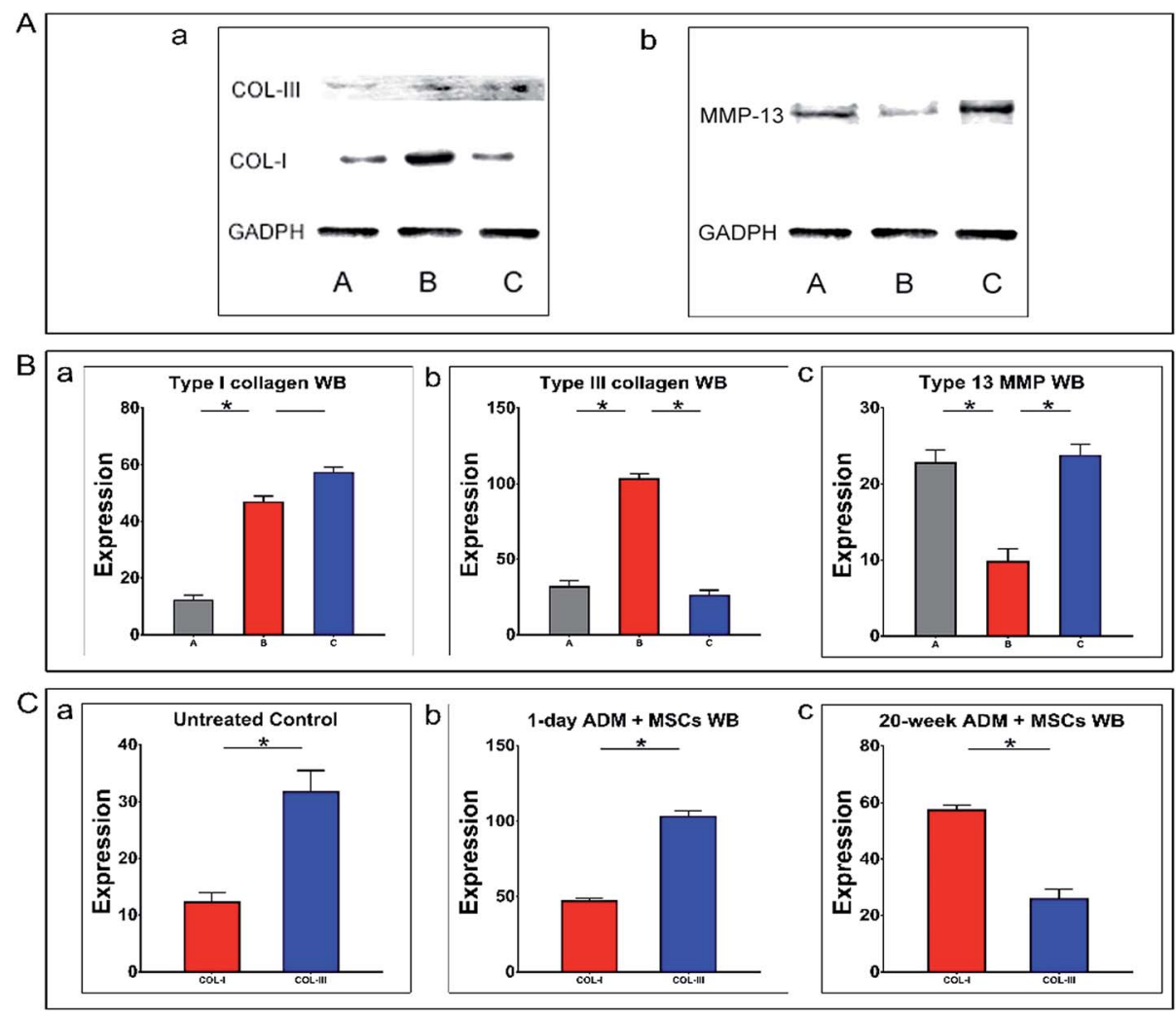

Fig. 7 Western blots comparing the translation of three indicated genes between experimental groups. The three indicated proteins were measured in the two experimental groups using GADPH as an internal reference. The expression of Col-I and Col-III proteins is shown in (A-a), and the expression of $\mathrm{Mmp}-13$ protein is shown in (A-b). As shown in panels (B-a) to (B-C), expression of the Col-III protein was up-regulated (Bb) and the Mmp-13 protein was downregulated $(B-C)$ in the 1 day ADM + MSCs group compared to the 20 week ADM + MSCs group. There was no significant difference in Col-I protein secretion (B-a). A statistical analysis of the within group expression of type I and type III collagens (C). In the 1 day ADM + MSCs group, more type III collagen than type I was present (C-b), and the converse was found in the 20 week ADM + MSCs group (C-C). Abbreviations: A: untreated control group. B: 1 day ADM + MSCs group. C: 20 week ADM + MSCs group. Col-I, type I collagen; ColIII, type III collagen; Mmp-13, type 13 matrix metalloproteinase; values shown are means \pm SD. $* p<0.05, * * p<0.01$.

imaging technique. TPF/SHG imaging is based on the optical emission signals of nonlinear optical effects produced by interactions of a near infrared femtosecond laser and biological tissues. These signals carry internal information about biological composition and microstructure. TPF is a two-photon absorption process and SHG is a coherent scattering process. This nonlinear optical imaging method provides unique imaging characteristics, including high image contrast, high spatial resolution, optical 3-D sectioning, minimal invasion, deep penetration and no interference from background light. ${ }^{26,27} \mathrm{~A}$ collagen fiber is one of the biological structures that produces strong intrinsic SHG signals. ${ }^{28}$ No extra dyes are added, thus avoiding toxicity and interference with the natural biological environment. TPF/SHG provides an ideal means for tracking MSC behaviors and monitoring collagen remodeling in the course of wound healing. In this study, 3-D images visualized the dynamic reconstruction of granulation tissue as well as the activities of the MSCs through layer-by-layer imaging of the newly formed granulation tissue. Our images demonstrated that after the transplantation of 1 day ADM + MSCs, the seeded MSCs migrated towards the wound bed more quickly and also showed a higher survival rate than MSCs in the 20 day transplants. Additionally, it was observed that the transplanted 1 day
ADM scaffold left less residual scaffolding but generated a thicker layer of granulation tissue than the 20 week ADM + MSCs group, which was considered to be the prior characterization of a better repair process. ${ }^{29}$

In wound repair with scar or scar-less tissue formation, studies indicated that they were associated with contents of different collagen types in granulation tissue.$^{30}$ In our study, immunofluorescent analysis of type I and type III collagen deposition in the granulation tissue revealed that in the 1 day ADM + MSCs group, the content ratio of type III/I collagen was higher than that in the 20 week ADM + MSCs group. These results were in accord with the results of a previous study, which demonstrated that scar-less wound repair in foetal skin produced high levels of type III collagen protein. ${ }^{31}$ The early appearance of type III collagen indicates an early increase in collagen synthesis that may provide wound structure and support for further necessary wound healing processes. ${ }^{32}$ In other words, 1 day ADM + MSCs treatment results in embryoniclike skin repair during the period of granulation tissue formation which, to our knowledge, generally leads to scar-less wound healing. Multiple factors participate in collagen synthesis and degradation, which regulate the formation of granulation tissue and change the collagen content. ${ }^{33}$ In our study, collagen 

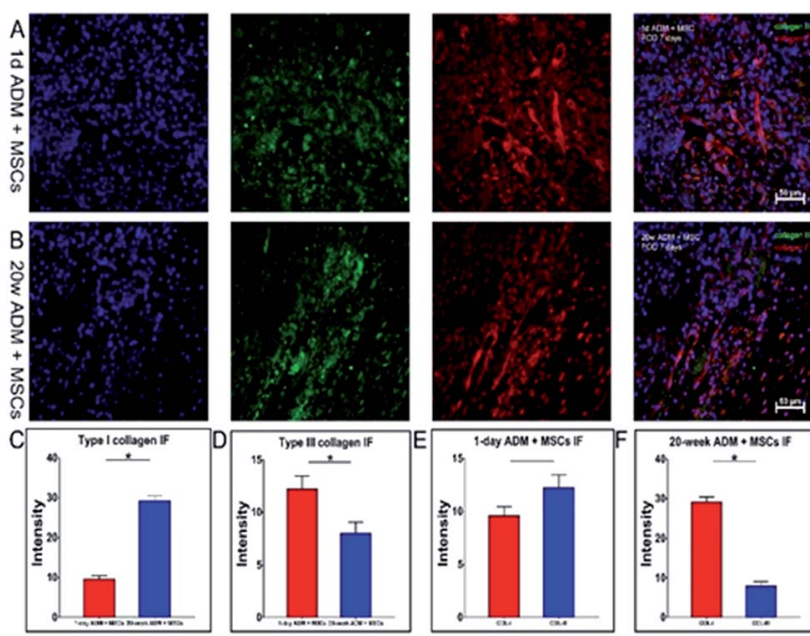

Fig. 8 Immunofluorescent analysis of the content of type I and type III collagens deposited in the granulation tissue. The two related collagens deposited in the granulation tissue were revealed by immunofluorescent staining (type I collagen: red and type III collagen: green) in (A and B). Statistical analysis of the immunofluorescence intensity of the two collagens. Less type I collagen (C) and more type III collagen (D) were deposited in the granulation tissue of the 1 day ADM + MSCs group compared to the 20 week ADM + MSCs group. A within group comparison revealed that in the 1 day ADM + MSCs group, the content of type III collagen was slightly higher than type I collagen (E), and in the 20 week ADM + MSCs group, there was more type I collagen deposited in the granulation tissue. (F) Abbreviations: Col-I, type I collagen; Col-III, type III collagen; values shown are means \pm SD. ${ }^{*} p<$ $0.05, * * p<0.01$. Scale bar $=50 \mu \mathrm{m}$.

synthesis was examined by assaying the expression of Col-I and Col-III, while collagen degradation was assessed by analysing the expression of matrix metalloprotease-13 (Mmp-13). RT-qPCR showed that Col-III expression was enhanced, and Western blotting demonstrated that the corresponding protein level was increased by treatment with 1 day ADM + MSCs. On the other hand, the expression of Mmp-13 in the 1 day ADM + MSCs group was found to be down-regulated. As predicted by these expression results, the deposition of type III collagen in granulation tissue in the 1 day ADM + MSCs group was higher than the deposition in the 20 week ADM + MSCs group. Similarly, the granulation tissue during foetal skin wound healing was found to have a higher Col-III/Col-I than the granulation tissue during adult skin wound healing. Hence, the wound repair promoted by 1 day $\mathrm{ADM}+\mathrm{MSCs}$ is predicted to be like the process that occurs in foetal skin.

The function of MSCs during wound healing is obvious. The addition of MSCs to 1 day ADM scaffolds enhanced the deposition of Col-III in granulation tissue relative to the deposition achieved by 1 day ADM scaffolds alone ${ }^{\mathbf{1 7}}$ and increased the Col$I I I / C o l-I$ in granulation tissue from 0.7 to 1.5 . Additionally, ADMs with different properties have been shown to have a disparate impact on collagen production. These phenomena remind us that MSCs participate in the granulation tissue rebuilding process, and their effects are regulated by the properties of the ADM. The factors and processes by which different ADMs induce such alterations remain to be further explored.
The ECM is a dynamic and complex environment characterized by biophysical, biomechanical and biochemical properties, which provides a dynamic niche for stem cells and regulates cell behaviours. ${ }^{34}$ Cells can sense their surrounding ECM microenvironment through focal adhesion that subsequently triggers a series of signalling pathways. ${ }^{35}$ Cell-ECM interactions not only involve the biochemical composition and structural organization of the ECM but also its mechanical properties. ${ }^{36}$ The mechanical properties of the ECM largely depend on three constituents, including elastic fibres, fibrillar collagens, glycosaminoglycans (GAGs) and proteoglycans (PGs), ${ }^{37}$ while collagen fibres endow the ECM with its material stiffness. Experiments demonstrated that MSCs can transmit biomechanical forces through actin-myosin contractions and differentiate into different types of cells based on the regulation of such external forces. ${ }^{38}$ Boris Hinz et al. found that as the ECM stiffness increased, the seeded MSCs were more likely to exhibit a myofibroblast phenotype. ${ }^{39}$ Myofibroblasts are modified fibroblasts with smooth muscle (SM)-like features, which were first observed in the granulation tissue of healing wounds. It is well-known that fibroblasts are the primary cells that synthesize collagen and secrete proteases; hence, they are the principle contributor to granulation tissue rebuilding and remodelling. ${ }^{\mathbf{4 0}}$ Fibroblasts were thought to determine the collagen characteristics of granulation tissue by generating contracted biomechanical force via a transitory myofibroblast phenotype. This force in turn influenced the secretory activity of fibroblasts to produce specific functional proteins such as type I collagen. ${ }^{\mathbf{4 1}}$ Matrix metalloproteinase families also play an important role in the growth of granulation tissue. ${ }^{42}$ In an $M m p-13$ knock-out animal model, the formation of granulation tissue was greatly enhanced. ${ }^{43}$ In our study, the ADM + MSCs group had lower matrix metalloproteinase-13 (Mmp-13) expression than the 20 week ADM + MSCs group, indicating that the 1 day ADM + MSCs may regulate granulation tissue formation via $M m p-13$ in a manner similar to the knockout. It is generally accepted that the biomechanical properties of the ECM could regulate the activity of matrix metalloproteinase through cell-ECM interactions. ${ }^{\mathbf{4 4}}$ Cells were shown to have maximal motility in an ECM environment exhibiting little stiffness. ${ }^{45}$ Enhanced migration of MSCs in the wound bed was also found in the 1 day ADM + MSCs group, which might be due to the regulation of matrix metalloproteinases via cell-ECM interactions. In our study, the differences in collagen type I and III production as well as the change in matrix metalloproteinase-13 ( $\mathrm{Mmp}-13)$ expression during granulation tissue formation might be the consequence of the remarkable difference in stiffness in the 1 day and 20 week ADMs, as shown in Fig. 2C-c.

There are other factors such as the composition, architecture or residual proteins such as fibronectin in the ADM that might cause differences in granulation tissue formation; ${ }^{46}$ however, as a result of our procedure for preparing the ADM scaffolds, most proteins, such as glycosaminoglycans, were removed, leaving only collagen and a natural structure. Therefore, the differences in the collagen content, architecture and biomechanics of the ADMs in the 1 day and 20 week groups are inferred to contribute to the differences in collagen synthesis and degradation during 
the formation of granulation tissue. Additionally, a study demonstrated that the stiffness manipulated independently of the gel architecture, polymer concentration, or adhesive ligand density of a wound dressing biomaterial dramatically affected the morphologies and spreading of fibroblast cells. In contrast, increasing the number of binding sites to which fibroblasts can adhere within a biomaterial does not subdue the mechanical stimuli that affect cell spreading and contraction. These results indicated that stiffness plays an independent and dominant role in fibroblast biology during the wound healing response, and other features were not highly relevant to fibroblast biology. ${ }^{47}$ Certainly, it is still too early to fully neglect the influence of other features of the ADMs, and it cannot be excluded that features such as composition or architecture might influence wound healing through regulating the stiffness of the ADMs.

\section{Conclusions}

Compared to 20 week ADM + MSCs treatment, 1 day ADM + MSCs treatment up-regulated the expression of Col-III and down-regulated the expression of Col-I and Mmp-13, resulting in a high Col-III/Col-I during granulation tissue formation, which is an indicator associated with the scar-less wound healing of foetal skin. Advanced nonlinear optical TPF/SHG imaging was applied to monitor the 3D interactions of MSCs with ADMs and the dynamics of the collagen rebuilding process in granulation tissue. One-day ADM + MSCs treatment also enhanced re-epithelization, angiogenesis and the generation of functional skin appendages. In conclusion, 1 day ADM biomaterial derived from neonatal skin can promote adult cutaneous wound healing and has the advantage of inducing embryoniclike skin repair.

\section{Acknowledgements}

This work was supported by the National Natural Science Foundation of China (No. 81671729) and (No. 81171379).

\section{References}

1 G. C. Gurtner, S. Werner, Y. Barrandon and M. T. Longaker, Nature, 2008, 453, 314-321.

2 P. Martin, Science, 1997, 276, 75-81.

3 S. H. Kim, J. Turnbull and S. Guimond, J. Endocrinol., 2011, 209, 139-151.

4 R. O. Hynes, Science, 2009, 326, 1216-1219.

5 D. E. Discher, D. J. Mooney and P. W. Zandstra, Science, 2009, 324, 1673-1677.

6 S. F. Badylak, Biomaterials, 2007, 28, 3587-3593.

7 H. K. Kleinman, D. Philp and M. P. Hoffman, Curr. Opin. Biotechnol., 2003, 14, 526-532.

8 C. C. Yates, R. Bodnar and A. Wells, Cell. Mol. Life Sci., 2011, 68, 1871-1881.

9 M. T. Longaker, D. J. Whitby, M. W. Ferguson, H. P. Lorenz, M. R. Harrison and N. S. Adzick, Ann. Surg., 1994, 219, 65-72.
10 A. S. Colwell, M. T. Longaker and H. P. Lorenz, Front Biosci., 2003, 8, s1240-s1248.

11 J. S. Choi, J. D. Kim, H. S. Yoon and Y. W. Cho, Tissue Eng., Part A, 2013, 19, 329-339.

12 N. A. Coolen, K. C. Schouten, E. Middelkoop and M. M. Ulrich, Arch. Dermatol. Res., 2010, 302, 47-55.

13 J. R. Merkel, B. R. Dipaolo, G. G. Hallock and D. C. Rice, Exp. Biol. Med., 1988, 187, 493-497.

14 B. M. Sicari, S. A. Johnson, B. F. Siu, P. M. Crapo, K. A. Daly, H. Jiang, et al., Biomaterials, 2012, 33, 5524-5533.

15 Q. N. Wang, Y. Jin, X. Y. Deng, H. P. Liu, H. W. Pang, P. P. Shi, et al., Biomaterials, 2014, 53, 659-668.

16 R. C. Rennert, M. Sorkin, R. K. Garg, M. Januszyk and G. C. Gurtner, Int. J. Biomater., 2013, 2013, 527957.

17 X. Han, H. Liu, M. Chen, L. Gong, H. Pang, X. Deng, et al., RSC Adv., 2016, 6, 71852-71862.

18 W. M. Jackson, L. J. Nesti and R. S. Tuan, Stem Cell Res. Ther., 2012, 3, 1-9.

19 F. M. Watt and W. T. Huck, Nat. Rev. Mol. Cell Biol., 2013, 14, 467-473.

20 G. Hosgood, Vet. Clin. North Am. Small Anim. Pract., 2006, 36, 667-685.

21 S. P. Huang, C. C. Hsu, S. C. Chang, C. H. Wang, S. C. Deng, N. T. Dai, et al., Ann. Plast. Surg., 2012, 69, 656-662.

22 M. C. Y. Heng, MD FRACP FACD FAAD, Int. J. Dermatol., 2011, 50, 1058-1066.

23 G. C. Gurtner, S. Werner, Y. Barrandon, et al., Nature, 2008, 453(7193), 314-321.

24 M. Jinnin, J. Dermatol., 2010, 37, 11-25.

25 R. K. Nath, M. Laregina, H. Markham, G. A. Ksander and P. M. Weeks, J. Pediatr. Surg., 1994, 29, 416-421.

26 M. Gu, X. Gan and X. Deng, Microscopic Imaging Through Turbid Media, Springer, Berlin Heidelberg, 2015.

27 W. R. Zipfel, R. M. Williams and W. W. Webb, Nat. Biotechnol., 2003, 21, 1369-1377.

28 E. Brown, T. Mckee, E. Ditomaso, A. Pluen, B. Seed, Y. Boucher, et al., Nat. Med., 2003, 9, 796-800.

29 L. Häkkinen, H. Larjava and L. Koivisto, Endod. Topics, 2011, 24, 125-173.

30 J. R. Merkel, B. R. Dipaolo, G. G. Hallock and D. C. Rice, Exp. Biol. Med., 1988, 187, 493-497.

31 M. J. Barnes, L. F. Morton, R. C. Bennett, A. J. Bailey and T. J. Sims, Biochem. J., 1976, 157, 263-266.

32 S. W. Volk, Y. Wang, E. A. Mauldin, K. W. Liechty and S. L. Adams, Cells Tissues Organs, 2011, 194, 25-37.

33 M. Xue, N. T. Le and C. J. Jackson, Expert Opin. Ther. Targets, 2006, 10, 143-155.

34 F. Gattazzo, A. Urciuolo and P. Bonaldo, Biochim. Biophys. Acta, 2014, 1840, 2506-2519.

35 B. Geiger, J. P. Spatz and A. D. Bershadsky, Nat. Rev. Mol. Cell Biol., 2009, 10, 21-33.

36 J. D. Humphrey, E. R. Dufresne and M. A. Schwartz, Nat. Rev. Mol. Cell Biol., 2014, 15, 802-812.

37 R. O. Hynes and A. Naba, Cold Spring Harbor Perspect. Biol., 2012, 4, a004903.

38 D. E. Discher, P. Janmey and Y. L. Wang, Science, 2005, 310, 1139-1143. 
39 B. Hinz, J. Biomech., 2009, 43, 146-155.

40 J. J. Tomasek, G. Gabbiani, B. Hinz, C. Chaponnier and R. A. Brown, Nat. Rev. Mol. Cell Biol., 2002, 3, 349-363.

41 F. Grinnell, Trends Cell Biol., 2000, 10, 362-365.

42 N. L. Boyd, S. S. Nunes, L. Krishnan, J. D. Jokinen, V. M. Ramakrishnan, A. R. Bugg, et al., Tissue Eng., Part A, 2013, 19, 211-223.

43 M. Toriseva, M. Laato, O. Carpén, S. T. Ruohonen, E. Savontaus, M. Inada, et al., PLoS One, 2012, 7, e42596.
44 R. J. Jerrell and A. Parekh, Acta Biomater., 2014, 10, 18861896.

45 M. H. Zaman, L. M. Trapani, A. L. Sieminski, D. Mackellar, H. Gong, R. D. Kamm, et al., Proc. Natl. Acad. Sci. U. S. A., 2006, 103, 10889-10894.

46 G. S. Schultz and A. Wysocki, Wound Repair Regen., 2009, 17, 153-162.

47 C. B. D. Cunha, D. D. Klumpers, W. A. Li, S. T. Koshy, J. C. Weaver, O. Chaudhuri, et al., Biomaterials, 2014, 35, 8927-8936. 